\title{
Probing the Dynamics of Non-Faradaic Processes in lonic Liquids at Extended Time and Length Scales Using XPS with AC Modulation
}

\author{
Pinar Aydogan Gokturk* and Sefik Suzer*
}

Cite This: J. Phys. Chem. C 2021, 125, 9453-9460

Read Online

ABSTRACT: Charging dynamics of ionic liquid (IL) electrolytes play important roles in various aspects of electrochemical processes. However, the precise understanding of such processes at extended time and length scales is incomplete due to the experimental difficulties in probing the electrochemical potential and other relevant parameters. In principle, such shortcomings should not apply to theoretical/computational approaches; however, existing works have mostly concentrated on or around electrode/electrolyte interfaces and short timescales due mostly to prohibitive demands on computational efforts. To fill this gap, we have utilized X-ray photoelectron spectroscopy to study the charging dynamics of ILs in contact with two wire electrodes under AC square wave excitation, with frequencies ranging from hundreds of $\mathrm{kHz}$ down to the $\mathrm{mHz}$ region. Using the changes in the binding energy position of the IL-related core-level peaks, electrical potential profiles along the lines in between the electrodes and on the entire surface of the electrolyte have been investigated in situ. From these results, we identify two widely different time constants. The timescale of the fast process was shown to be on the order of $R C$ time constant, while the slow process takes place on a timescale of seconds. Our method in the present study is expected to open up a new way for extracting novel dynamic information for gaining a better understanding of such processes and designing efficient IL-based electrochemical devices with a novel perspective on the charging.

\section{INTRODUCTION}

Non-Faradaic processes, particularly the electrochemical double-layer (EDL) dynamics, play a key role in the electrochemistry of electrified interfaces because when an electrolyte solution comes into contact with a polarized/charged surface, ions at the interface rearrange to screen this charge. ${ }^{1}$ As a result, a concentration and, therefore, a potential profile is formed at the electrode/electrolyte interface, which governs the chargetransfer processes and influences the kinetics and thermodynamics of the electrochemical systems. ${ }^{2}$ That is why many attempts have concentrated on the investigation of the EDL properties, both experimentally and theoretically. Until now, numerous theories and models have been developed to describe the structure of ions at the electrode/electrolyte interfaces of dilute solutions, ${ }^{1,3,4}$ yet a detailed understanding of the EDL processes of more complex and high-concentration liquids such as room-temperature ionic liquids (RTILs) remains elusive, due especially to the lacking of solvent molecules, ion size, and steric effects. $^{5-10}$

RTILs are molten salts composed of large organic cations and either organic or inorganic anions. Properties such as the high ionic strength, large electrochemical window, high capacitance, low volatility, and so forth make ionic liquids (ILs) a good candidate for the choice of electrolyte for next-generation energy storage and conversion devices. ${ }^{11-15}$ The same properties also result in various phenomena unique to IL EDLs, such as a multilayer interfacial structure, overscreening, crowding, and differential capacitance profiles, which were not observed for dilute solutions. ${ }^{6,9,16}$ Although there has been great interest in ILs and their EDL properties, a majority of previous studies on IL EDLs so far have focused on the steady-state properties at equilibrium. ${ }^{7,17-19}$ Therefore, the dynamic aspect of how the potential and ion structure evolve with time has been explored at a much slower pace.

The dynamics of the EDL is directly related to the timedependent properties of electrochemical devices, such as the power density and the charging/discharging rate. Thus, it is necessary to investigate the evolution of the EDL during the charging and discharging processes for gaining a better understanding of such devices. Once a potential is applied to the electrode, mainly two driving forces, migration and diffusion, govern the EDL dynamics. First, ions migrate to the electrode/ electrolyte interfaces in order to sustain the local electroneutrality. The time that ions take to migrate is often referred to as the Debye time, which is a function of EDL thickness and ion diffusivity in the particular electrolyte. ${ }^{1}$ The migration of ions

Received: February 11, 2021

Revised: March 20, 2021

Published: April 22, 2021 
a)

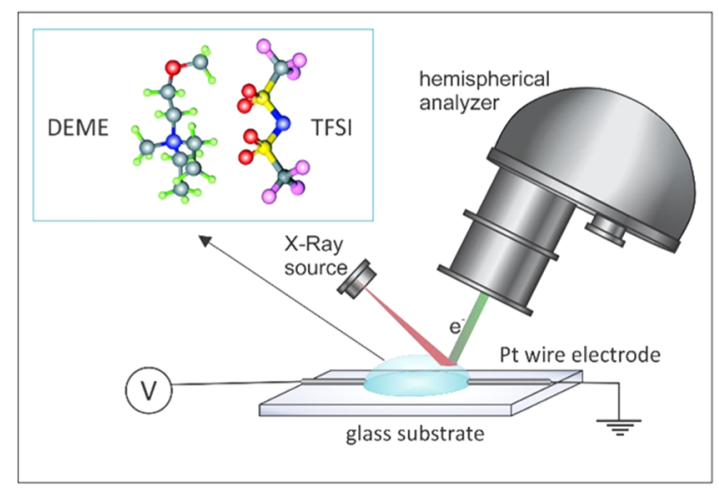

b)

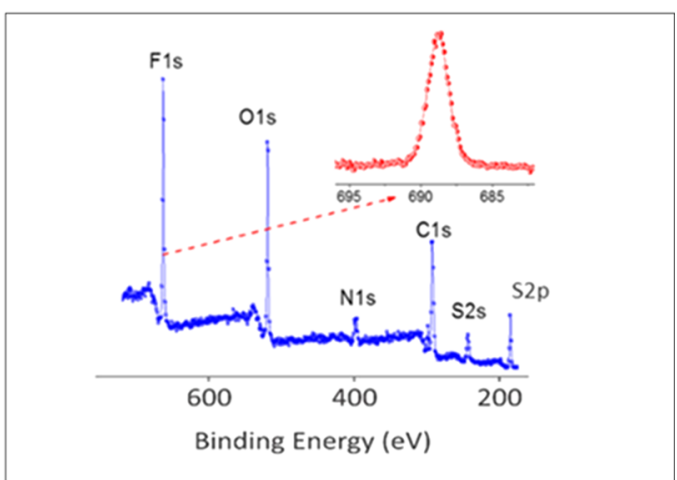

c)

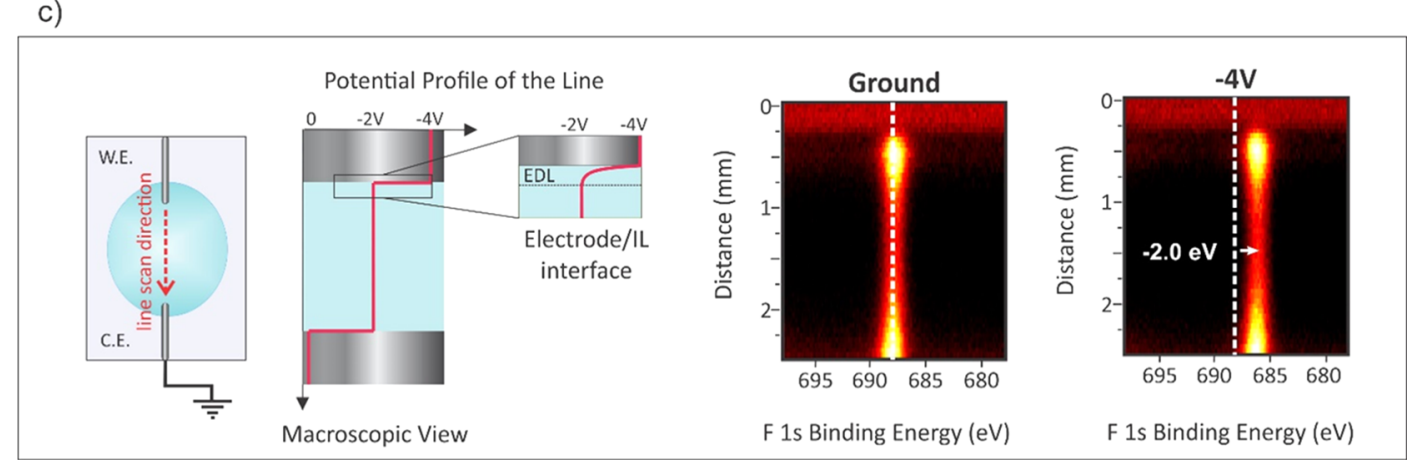

Figure 1. (a) Schematization of the experimental setup for probing the dynamics of the EDL of ILs by XPS under operando conditions. The function generator is connected to one of the electrodes (WE) to apply a DC or AC SQW excitation with a fixed or variable frequency, while the other one (CE) is grounded. Inset shows the chemical structure of the IL used in this study. (b) Survey XP spectrum of the IL and the F 1s region recorded in the scanning mode. (c) F 1s line spectra recorded when the WE is grounded and $-4 \mathrm{~V}$ DC bias is applied using the 1s snapshot mode. Schematics of the electrochemical cell showing the line scan direction and the corresponding potential profile detectable throughout the bulk IL electrolyte under the -4 V DC bias is also shown.

results in changes in ion concentrations and a potential gradient away from the electrode surface, which then induce the diffusion of ions from the bulk electrolyte toward the interface region. Diffusion is a much slower process and takes place on the timescale which additionally depends on the geometry of the electrochemical system, specifically the distance between electrodes. ${ }^{1}$ Theoretically, it was previously shown that the classical Poisson-Nernst-Planck (PNP) equation cannot accurately predict the EDL properties for ILs because it neglects the ion size and steric effects. ${ }^{5}$ Moreover, for ILs, the traditional time constants used for dilute electrolytes are not applicable either. That is why the PNP equation has been further modified using a continuum model to take steric effects into account. ${ }^{20-22}$ This modified model has been shown to be applicable to concentrated electrolytes as well as ILs and molten salts. Using such a model, overscreening and crowding phenomena were successfully modeled for IL EDLs. Accordingly, new time and length scales were identified for the charging of a parallel-plate electrochemical cell. This new timescale was also shown to be consistent with the classical resistor-capacitor $(R C)$ circuit model under certain circumstances. ${ }^{6}$

On the experimental side, previous studies, to understand the formation and relaxation of EDLs, have mostly focused on the electrical characterization techniques such as cyclic voltammetry, chronoamperometry, and electrochemical impedance spectroscopy (EIS). ${ }^{23-25}$ EIS is one of the most commonly used technique to explore the charging dynamics of ILs due to its ability to differentiate different components within devices (decoupling of resistive and capacitive components) from their frequency-dependent response to the imposed potential. EIS measurements previously revealed the presence of two distinct capacitive processes taking place on different timescales at the IL/gold electrode interfaces. The timescale of the fast process was shown to be typically on the order of $R C$ time constant of milliseconds, while the slow process takes place on a timescale of seconds. ${ }^{26}$ Slow capacitive and other processes have also been shown for other IL/electrode interfaces. ${ }^{27,28}$ Although electrical characterization techniques are helpful in identifying the time dependence, they are unable to explore the spatial variations in the potential to interrogate local properties, and they cannot identify the nanoscale origins of the time dependence. There have also been a number of other experimental studies showing interfacial dynamics in ILs. For instance, Uysal et al. performed an X-ray reflectivity study on the IL/graphene interface and discovered a slow time constant $>1 \mathrm{~s}$, much slower than the $R C$ timescale. ${ }^{29}$ This finding was supported in a more recent study using the same technique, where the presence of an ultraslow dynamics at a charged silicon-ionic liquid interface was reported. ${ }^{30}$ The data reported also illustrated that crowding of anions has timescales in the order of $\sim 10^{3} \mathrm{~s}$. However, a complete understanding of the time response of such processes would require techniques capable of mapping the potentials or concentration of ions not only at the electrode/electrolyte interface but also throughout the entire liquid. Recent attempts using electrochemical force microscopy have shed intriguing new inputs into the dynamics of charge screening at the solidaqueous interfaces by identifying multiple time constants and relatively larger length scales of up to $10 \mu \mathrm{m} .{ }^{31,32}$ However, 
again, such distances are still small to represent real-life electrochemical devices. In order to fill this gap, we introduce $\mathrm{X}$-ray photoelectron spectroscopy (XPS) with AC modulation.

XPS enables quantitative analysis with chemical specificity and surface sensitivity. A facile advantage of XPS to tap into the local potential information makes it also suitable for investigating various electrochemical systems. Under an external electrical excitation, core-level photoelectrons measured by XPS reveal binding energy shifts that reflect the local potential $(V)$ on the surface by $\Delta \mathrm{BE}=\Delta \mathrm{VeV}$, in a chemically specific and relatively noninvasive fashion. Previously, tender ambient pressure XPS with a dip-and-pull configuration was used to probe the shape of EDL at the solid/liquid interface of aqueous electrolytes as a function of the concentration and applied potential. ${ }^{10}$ Similarly, in one of the first studies of ILs, a drop of IL was placed on an angled electrode. This experimental setup allowed the formation of a liquid film thin enough to probe the $\mathrm{IL} /$ electrode interface, so that the authors were able to follow the EDL processes by tracing the shifts in binding energy under an external potential. ${ }^{33}$ While applying such DC potential during XPS data acquisition provides vital steady-state information, our group has previously shown that $\mathrm{AC}$ excitation is helpful for understanding the dynamics of electrical potential developments. $^{34-39}$

In this study, we report direct probing of the diffuse-charge dynamics of the IL $N, N$-diethyl- $N$-methyl- $N$-(2-methoxyethyl)ammonium bis(trifluoromethylsulfonyl)imide (DEME-TFSI) by means of XPS under AC potential excitation, with frequencies ranging from $\mathrm{mHz}$ to $\mathrm{kHz}$ at room temperature. For such analysis, areal and line scan modes at different but constant frequencies are employed to provide spatial information from extended length scales. Additionally, time-resolved XPS with a fast data collection mode having 1 s resolution allowed us to follow the temporal evolution of the electrical potential on the surface by the continuous collection of spectra as a function of time, while exposing the surface to AC square wave (SQW) excitation sweeping with a logarithmic frequency change. ${ }^{36}$ This kind of direct measurement of potential development dynamics on devices, at the position where and while they occur, has not heretofore been possible. From the spectral results, we extracted two different time constants. By considering the geometry of our electrochemical cell, it was also possible to estimate the diffusion constant for IL, which was found to be comparable with previous reports. In addition, an asymmetric behavior of the system with respect to the polarity, under both DC and AC excitations, was also discovered, and the possible origins of this behavior were elaborated. We believe that the findings from this study provide direct insights into the charging dynamics of IL-based electrosystems at extended time and length scales while also demonstrating the utility of XPS with electrical potential sensitivity under AC excitations.

\section{METHODS}

Materials. DEME-TFSI was purchased from Sigma-Aldrich with purity $\geq 98.5 \%$ and used without any purification.

Preparation of Electrochemical Devices. The device configuration used in this study is shown Figure 1a. In this configuration, two Pt wires were used as electrodes, and $5 \mu \mathrm{L}$ of IL (DEME-TFSI) was injected to the substrate between the electrodes. One of the electrodes was used as the working electrode (WE) to apply the external bias, while the other one, the counter electrode (CE), was kept at ground potential.
XPS Measurements. All XPS measurements were conducted with a Thermo Fisher $\mathrm{K} \alpha \mathrm{X}$-ray photoelectron spectrometer having monochromatized X-rays of $1486.6 \mathrm{eV}$. All core-level spectra were collected using $50 \mathrm{eV}$ analyzer pass energy. The X-ray spot size was set to $50 \mu \mathrm{m}$ for areal and line scan measurements and $100 \mu \mathrm{m}$ for single-point measurements. For time-resolved XPS, the fast data gathering, the snapshot mode with $150 \mathrm{eV}$ pass energy, was used instead of the scanning mode. The pressure in the analyzing chamber was always kept under $10^{-8} \mathrm{mbar}$. The fitting procedure was performed using the Avantage Software equipped with the instrument. All fits reported in this work have been carried out using a symmetrical Gaussian/Lorentzian product function with ratios of 30/70 after Shirley background subtraction.

\section{RESULTS}

We selected DEME-TFSI as the IL because DEME cations have a relatively large electrochemical window around $\sim 7-8 \mathrm{~V}$, which enables us to apply a larger magnitude of external potentials. ${ }^{40}$ XPS measurements were performed on the IL drop confined between two Pt electrodes (see Figure 1a). The function generator is connected to the $\mathrm{WE}$, while the $\mathrm{CE}$ is grounded. Either a DC or an AC signal in the form of SQWs is generated and applied with a fixed or sweeping frequency depending on the mode of data collection. The SQW modulation, rather than the conventional sine wave, is adopted as it introduces the least amount of distortion to the line shapes of the XPS signals. Details of the experimental methods are given in the Supporting Information. In order to follow only the EDLrelated processes, the amplitude of the external bias was chosen such that the Faradaic sources and possible redox reactions do not contribute to the spectral features during the experiment, but ion transport dynamics are facilitated throughout the relatively short measurement times within a few minutes. In our previous studies, we were able to identify and probe in situ redox reactions of different ILs that are imidazolium-based to form stable carbenes but only after prolonged DC excitations, up to several hours. ${ }^{41,42}$ However, for the current study, the presence of new species or chemistries was not observed during the measurements; hence, we assume that the electrodes are ideally polarizable without significant Faradaic reactions, with the steady-state currents on the order of $\sim 10 \mathrm{nA}$ passing through the IL drop. Accordingly, our electrochemical cell can be considered as a parallel-plate capacitor with blocking electrodes. A survey spectrum of the IL and the recorded F 1s (representing the IL) core-level XP spectra when the WE is grounded and under DC excitation are shown in Figure 1b,c.

Considering the two symmetrical metal contacts without a significant contribution of Faradaic reactions, at very low frequencies and DC excitations, ideally equal amounts of potential drops at the electrode/IL interfaces through the formation of two complementary double layers are expected to materialize. Therefore, only half of the imposed potential is expected to be measurable as shifts in the binding energy throughout the surface of the bulk IL (see Figure 1c). Similar observations were also obtained in our previous study. ${ }^{34}$ Note that the experimentally available lateral/spatial resolution of our instrument $(\geq 50 \mu \mathrm{m})$ is not small enough to capture any direct information related with the EDLs at the interface, but indirect observation can be done by following the potential in the bulk IL electrolyte (Figure 1c). Our measurements reveal a slight asymmetry: $+2.5 \pm 0.1$ and $-2.0 \pm 0.1 \mathrm{eV}$ shifts, under +4 and $-4 \mathrm{~V} \mathrm{DC}$ bias, respectively, as reproduced in the data shown in 
a)

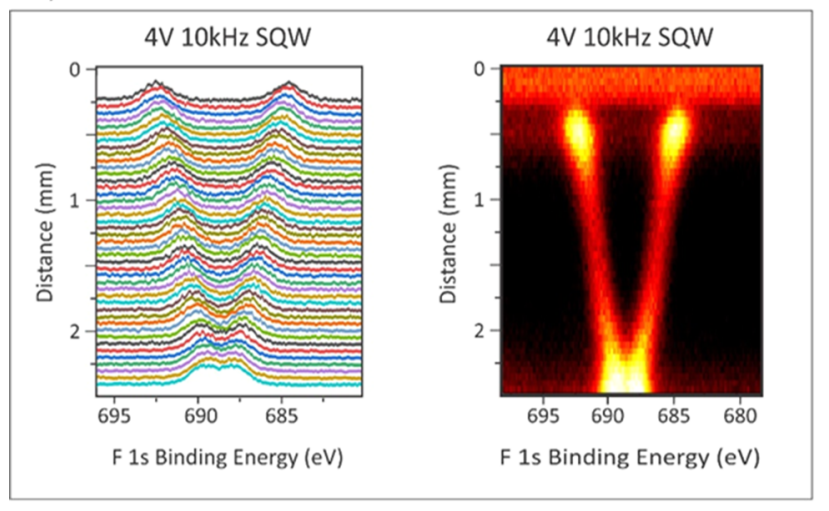

b)

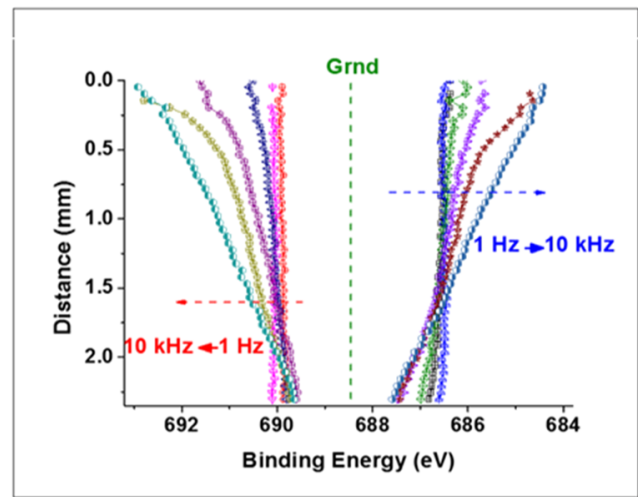

C)

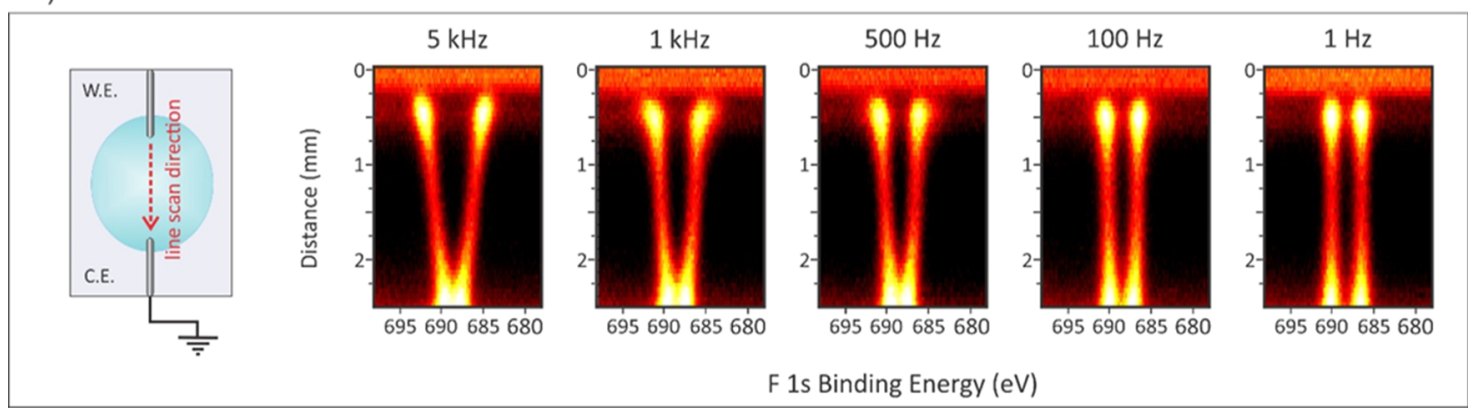

Figure 2. (a) Two different representations of F 1s spectra recorded under $4 \mathrm{~V}$ SQW AC excitation with $10 \mathrm{kHz}$ frequency along the designated line. (b) Extracted binding energy positions of the F 1s peaks at both positive and negative cycles of six different frequencies. (c) F $1 \mathrm{~s} \mathrm{spectra}$ recorded under $4 \mathrm{~V} \mathrm{SQW}$ excitation along the line as designated at five different but fixed frequencies, together with the schematic representation of the electrochemical cell.

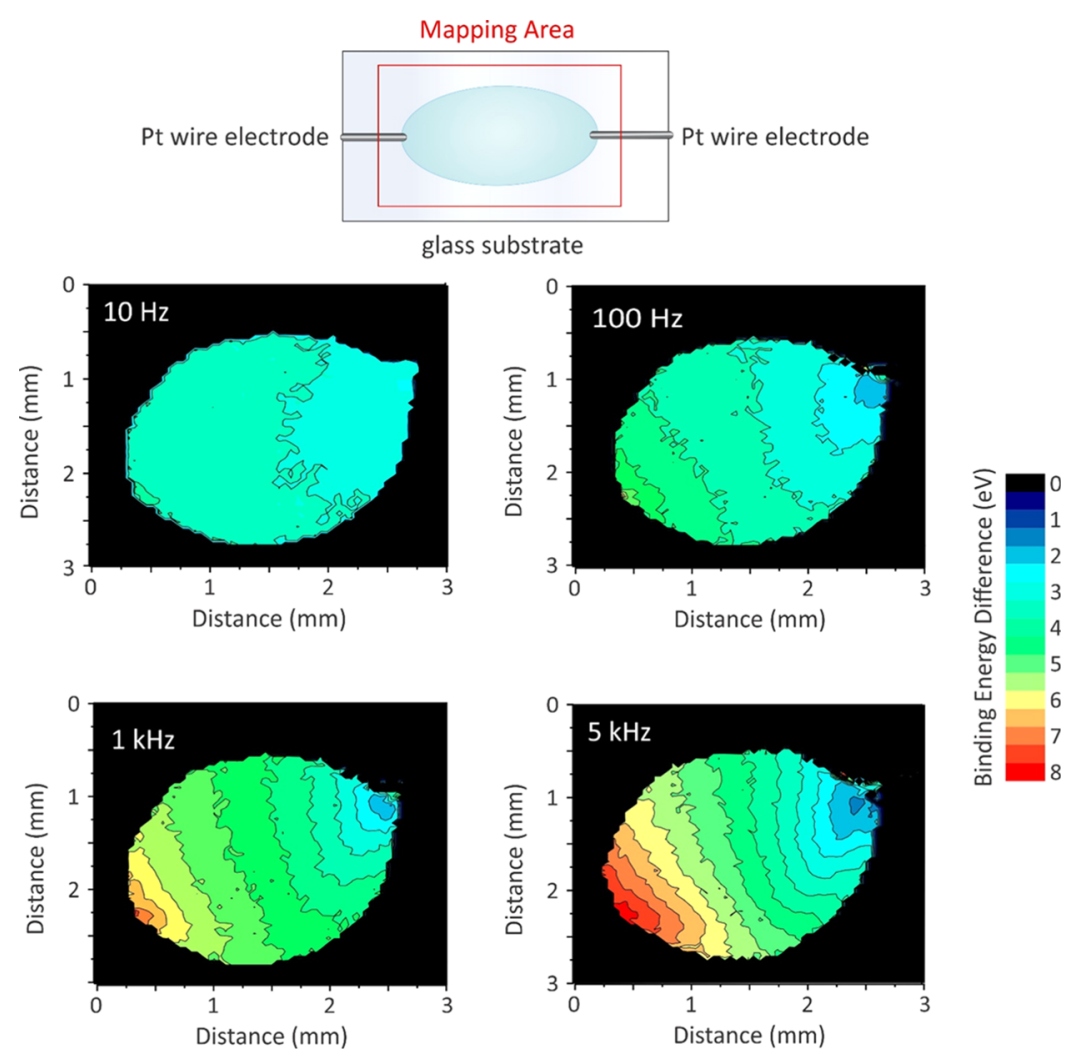

Figure 3. Areal maps of the binding energy difference of the positive and negative components of $\mathrm{F} 1 \mathrm{~s}$ under the $\mathrm{SQW}$ excitation with $10 \mathrm{~Hz}, 100 \mathrm{~Hz}, 1$ $\mathrm{kHz}$, and $5 \mathrm{kHz}$ frequencies, together with the pictorial representation of the experimental setup and mapping area used for the measurements. 

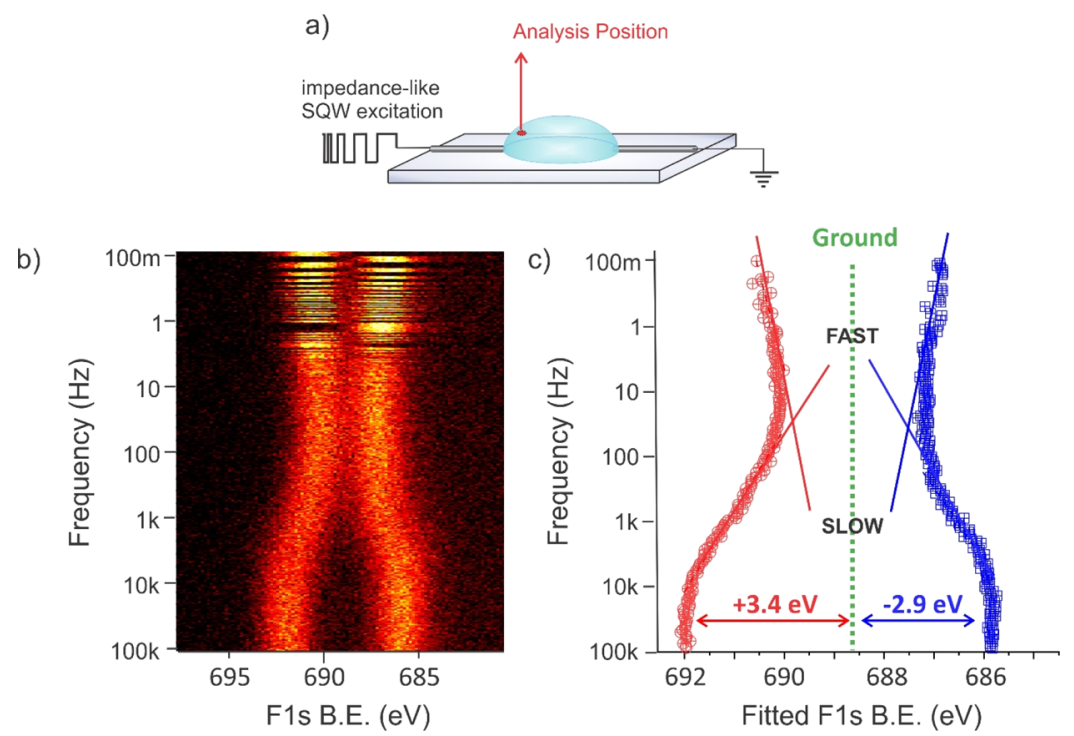

Figure 4. (a) Schematics of the application of a continuous logarithmic AC frequency sweep and the point at which the data are recorded. (b) F 1s XP spectra recorded under a continuous $4 \mathrm{~V} \mathrm{SQW}$ logarithmic frequency sweep in the range of $0.1 \mathrm{~Hz}$ to $100 \mathrm{kHz}$. (c) Extracted binding energy positions in the positive (red) and negative (blue) cycles of the SQW excitation, emphasizing once more on the asymmetric behavior of the device.

Figure S3. This small but measurable asymmetry under DC excitation is also evident under AC actuation, as will be discussed later.

In order to capture the temporal evolution of positiondependent potential developments on the electrochemical cell consisting of the DEME-TFSI electrolyte, we collected the F 1s (coming from the liquid) core-level spectra along a selected line starting from the WE toward the grounded CE. During the data acquisition, an external AC bias in the form of SQWs with the amplitude of $8 \mathrm{~V}$ [peak-to-peak voltage $\left(V_{\mathrm{p}-\mathrm{p}}=8 \mathrm{~V}\right)$ ] is applied. The two-dimensional (2D) image plots of F 1s XP spectra and the extracted binding energy positions are shown in Figure 2 as a function of interelectrode distance at various but fixed frequencies. For a better understanding, the corresponding XP spectra at $10 \mathrm{kHz} \mathrm{AC}$ excitation are displayed in two different (stacked and 2D image) fashions in Figure 2a. As can be gathered from Figure $2 a, c$, at high frequencies, the entire potential drops linearly across the bulk electrolyte as the ions do not have enough time to screen the bias properly before switching the polarity, whereas as the frequency is lowered, the potential starts to be screened due to the ionic rearrangement around the vicinity of the electrodes (formation of EDL). The derived electrical potential information reflects an average over the ensemble of IL molecules within the selected X-ray spot size. Although the experimentally available lateral resolution of our instrument is not enough to capture the EDL region, the deviation of the potential profiles from linearity, especially at the vicinity of the $\mathrm{WE} /$ electrolyte interface is still visible at distances which are far beyond the width of the double layer (Figure $2 b$ ).

To extend these measurements to the entire droplet surface, we have recorded the $\mathrm{F} 1 \mathrm{~s}$ signal in the areal mapping mode and at various frequencies. We then fitted the XP spectrum at each point to two $\mathrm{F}$ 1s peaks, corresponding to the negative and positive components of the SQW signal, and measured the binding energy difference between them. The binding energy differences of the two components from the entire area are displayed in Figure 3 as areal maps for each frequency. Details of the data handling are given in the Supporting Information. Such $2 \mathrm{D}$ areal recording during XPS data acquisition is useful to visualize the local potentials across the electrochemical cell and generate equipotential-like contour plots. Note that the "roughness" in the equipotential lines is solely an artificial observation coming from the choice of the potential interval. The pixelated representation of contour plots (capturing our spatial resolution) with various number of major color levels is provided for the spectra taken under SQW excitation with a frequency of $5 \mathrm{kHz}$ in Figure S4.

To accurately capture the frequency-dependent transition of the IL from the complete screening to resistive behavior, we applied a time-varying SQW electrical signal with a logarithmic frequency sweep, starting from $100 \mathrm{mHz}$ and reaching $100 \mathrm{kHz}$ with an amplitude of $4 \mathrm{~V}$, instead of distinct frequencies. We then followed the surface potentials by recording the $\mathrm{F} 1 \mathrm{~s}$ signal at a position close to the WE (see Figure 4). The most relevant electrical information derivable from such a measurement is the cutoff frequency at which the transition of the IL medium from the shielded to resistive behavior takes place.

Frequency response curve of the binding energy in Figure 4 resembles the well-known high-pass filter where the signal is attenuated at low frequencies, whereas the output signal increases with the increasing frequency until it reaches a steady-state value. However, contrary to the typical high-pass filters, the input signal is a SQW, where both the shape and the magnitude of the output response depend drastically on the circuit's $R C$ time constant and input frequency. In addition, a much slower process associated with frequency lower than $1 \mathrm{~Hz}$ is observable in the low-frequency end of the F 1s binding energy response curve (Figure 4c). Although our instrumentation limits the precise estimation of this time constant, this is still faster than the one for diffusion across the macroscopic length of the cell. With a closer look, the asymmetric behavior in between the negative and positive components of the SQW signal can also be captured from the fitted results of F 1s binding energy (Figure 4c).

\section{DISCUSSION}

In electrochemistry, an important approach is the construction of an equivalent electrical circuit, whose parameters fit to the 
a)

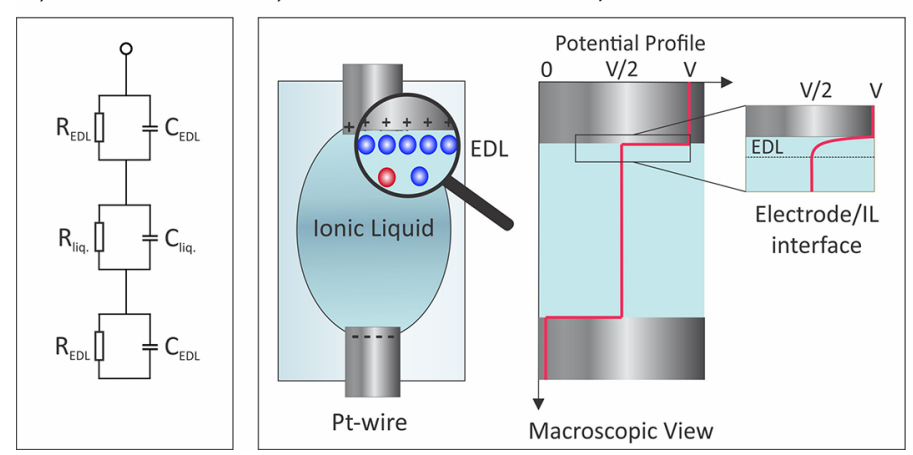

c)

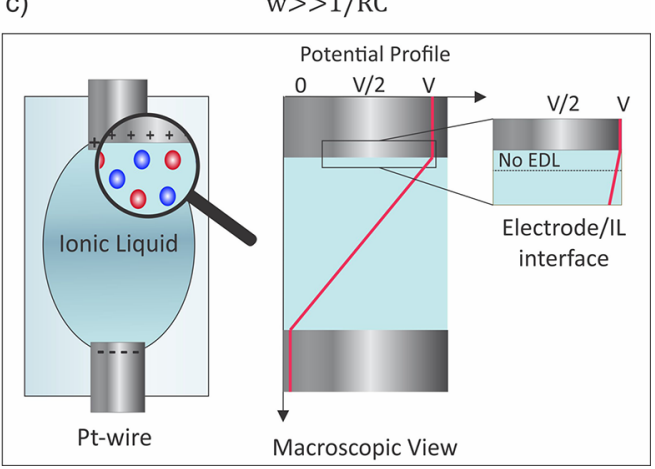

Figure 5. (a) Equivalent electrical circuit representing the simple two-electrode electrochemical cell. A schematic representation of the formation of the EDL in the electrochemical cell under an AC potential bias and the respective potential profiles inside the cell for the excitation frequency: (b) much lower and (c) much higher than the $R C$ time constant. For lower frequencies, the mobile ions move toward the electrode and form an EDL at the two interfaces. Note that these pictorial descriptions are not intended to show the accurate length scales of EDL thickness or the structure of ion rearrangements; they merely represent the time-dependent changes in the potential profiles observed in the bulk electrolyte.

experimental response(s). Similar approach can also be adopted to understand and rationalize the experimental XPS data given in Figures 2 and 3. For a simple electrochemical cell consisting of a liquid electrolyte between two symmetrical electrodes, as shown in Figure 5, the liquid electrolyte can be modeled as a resistor, while the two electrical double layers formed at the IL/electrode interfaces are represented by a parallel resistor and a series capacitor. In the $R C$ circuit theory, the characteristic time of response of a device is represented by $\tau=R C$, also known as $R C$ time constant, where $R$ is the effective resistance and $C$ is the total capacitance. When current passes through an electrolyte between two electrodes, and for the frequency $w \ll 1 / R C$, all the voltage is dropped across the EDLs. Similarly, for the frequency, $w \gg 1 / R C$, all the voltage is dropped across the electrolyte. The reason for this is, at high frequencies, ionic movements are basically frozen and ions do not have enough time to rearrange and form a double layer, whereas at lower frequencies, ion transport facilitates the formation of the EDL; hence, a nonchanging half of the applied potential appears throughout the entire electrolyte medium in between the electrodes.

If the applied potential is sinusoidal, at a frequency equal to 1 / $\tau$, half of the potential drops across the EDLs and the other half across the electrolyte. This $1: 1$ ratio changes to $0.63: 0.37$ if the input is SQW instead of sine wave. ${ }^{43}$ Although the $R C$ circuit model fails to capture the spatial dependence of the voltage, it can still be applied to estimate the time constant of our system. Our instrumental timescale of data acquisition cannot capture the real-time potentials at high frequencies, and one should also remember that the observed shift in the binding energy is averaged over the time window of data collection. As a result, the shape of the output waveform depends on both the frequency of the input SQW and the $R C$ time constant of the circuit. When the frequency of the input is lower than the $R C$ time constant, the output waveform resembles the SQW, whereas the higher frequencies produce output waveforms as narrow positive and negative spikes (illustration of the output waveforms at different frequencies for the equivalent electrical circuit of the electrochemical cell is given in the Supporting Information). Knowing that $0.63 \mathrm{~V}$ of potential drops across the EDLs at the frequency equal to the $R C$ time constant and considering the total magnitude of the external AC bias in the form of SQWs $\left(V_{(\mathrm{p}-\mathrm{p})}=\right.$ $8 \mathrm{~V}$ ), the frequency equal to $1 / \tau$ should yield $\sim 3 \mathrm{~V}$ potential drop across the electrolyte. Therefore, this frequency (time constant) can easily be estimated to be around $100 \mathrm{~Hz}(\tau=0.01$ s) from Figures 2 and 3.

Similarly, the $R C$ time constant $[\sim 100 \mathrm{~Hz}(\tau=0.01 \mathrm{~s})]$ can also be determined by fitting the frequency response curve in Figure 4. It is well known that, for the pulse width (half of the period for the output waveform) equal to the $R C$ time constant, the output spike discharges down to $37 \%$ of its initial value. ${ }^{44}$ An equivalent electrical circuit of our electrochemical cell and the corresponding output waveforms are given in Figure S5.

With the careful evaluation of data, small differences in the frequency response of the negative and positive components of the SQW can also be identified. The possible sources of this asymmetry may be attributed to the difference(s) in (i) the capacitance of the metal electrodes due to the unintentional geometrical forms/shapes of the two electrodes ${ }^{38}$ and the other one; (ii) the mobility or the diffusion coefficient of the cations and the anions, as advocated in a recent paper by Greco et al. ${ }^{45}$ The third possibility is, of course, the presence of some unavoidable Faradaic processes. Interestingly, the frequency response curve reveals another and much slower time constant associated with frequencies lower than $1 \mathrm{~Hz}(\tau>1 \mathrm{~s}$ ) (see Figure 4c).

Although there is no generally accepted theory of ion dynamics in an RTIL, as noted earlier in the Introduction section, there are several theoretical predictions. The three obvious timescales reported in the literature ${ }^{5}$ are $\tau_{D}=l_{d}^{2} / D, \tau_{c}=$ $l_{d} L / D$, and $\tau_{L}=L^{2} / D$, where $l_{d}$ is the Debye length, $2 L$ is the distance between the electrodes, and $D$ is the diffusion constant. These are the time constants associated with the local charge redistribution (EDL), diffusion across the cell, and diffusecharge dynamics, respectively. The typical experimentally observed Debye length is of the order of $1-10 \mathrm{~nm}^{46-48}$ Similarly, the typical diffusion constant for similar ILs is around $10^{-10}$ to $10^{-11} \mathrm{~m}^{2} / \mathrm{s}^{26,49-51}$ Taking $L \cong 1 \mathrm{~mm}$ as in our electrochemical cell, $D=5 \times 10^{-11} \mathrm{~m}^{2} / \mathrm{s}$, and $l_{d}=2.5 \mathrm{~nm}$, we obtain $\tau_{D} \cong 1.2 \times 10^{-7} \mathrm{~s}, \tau_{\mathrm{c}} \cong 0.05 \mathrm{~s}$, and $\tau_{L} \cong 2 \times 10^{3} \mathrm{~s}$. Compared to our results, $\tau_{D}$ is far too small. Hence, the experimentally measured time constant should be equal to the time constant for diffuse-charge dynamics, which is expected to dominate the transient response, as proposed by Bazant et al. ${ }^{5}$ Although our experimentally measured time constant for diffuse-charge dynamics is close to the prediction, the much slower time constant that is observable in Figure 4 is hard to be associated with $\tau_{L}$. 
It should be pointed out that these theoretical timescales are only true for one-dimensional two-electrode electrochemical cell models under negligible inertial effects, identical diffusivities of cations and anions, and small electric fields. Additionally, the usual assumptions for this type of modeling break down for high voltage or high ionic strength. Even though we intended to create a one-dimensional geometry, ILs on glass substrates form a certain contact angle due to low wettability. The secondary slower time constant we observed may arise from the geometrical factors associated with the three-dimensional shape of our electrochemical system. Such argument was the reason for advocating that, for a cylindrical pore, $\tau_{\mathrm{c}}$ should be modified by a geometrical factor. ${ }^{52,53}$

\section{CONCLUSIONS}

In this work, the diffuse-charge dynamics of an IL-based electrochemical cell were probed in situ using XPS under AC SQW excitation. Spectral data revealed that the time constant for the diffuse-charge dynamics of DEME-TFSI is around $0.01 \mathrm{~s}$. To our knowledge, this kind of direct measurement of the potential development dynamics at the position where and while they occur has not heretofore been reported. Unlike the traditional electrochemical methods generally employed to measure the time constants and dynamics of these non-Faradaic processes, XPS provides a chemically specific electrical potential information, with the spatial resolution determined by the instrument, but in theoretically unlimited length scales. This feature can be extremely handy for detecting roadblocks, hence enabling reliable device performance checks on real-life systems. In general, we believe that the ability to directly observe the time-dependent potential developments on the entire electrosystem facilitates precise understanding and eventually helps to drive the design of next-generation devices.

\section{ASSOCIATED CONTENT}

\section{SI Supporting Information}

The Supporting Information is available free of charge at https://pubs.acs.org/doi/10.1021/acs.jpcc.1c01292.

XP spectra under DC and AC excitation; F 1s line spectra recorded under $+4,0$, and $-4 \mathrm{~V} \mathrm{DC}$ bias; peak fitting of areal maps and constructing $2 \mathrm{D}$ contour plots of potential distribution; areal maps of the binding energy difference of the positive and negative components of $F$ 1s under the SQW excitation with $5 \mathrm{kHz}$ frequency; and output waveforms at different frequencies for the equivalent electrical circuit of the electrochemical cell (PDF)

\section{AUTHOR INFORMATION}

\section{Corresponding Authors}

Pinar Aydogan Gokturk - Department of Chemistry, Bilkent University, Ankara 06800, Turkey; 이이.org/0000-00018269-8884; Email: paydogan@lbl.gov

Sefik Suzer - Department of Chemistry, Bilkent University, Ankara 06800, Turkey; (i) orcid.org/0000-0002-5866-

2600; Email: suzer@fen.bilkent.edu.tr

Complete contact information is available at:

https://pubs.acs.org/10.1021/acs.jpcc.1c01292

\section{Author Contributions}

The manuscript was written with contributions of both authors. P.A.G. planned and executed the experiments, evaluated the data, and drafted the manuscript. S.S. supervised the study, planned the experiments, and drafted the manuscript.

\section{Notes}

The authors declare no competing financial interest.

\section{ACKNOWLEDGMENTS}

This work was supported by grant no: $118 \mathrm{Z} 902$ of the Scientific and Technological Research Council of Turkey (TUBITAK).

\section{REFERENCES}

(1) Bard, A. J.; Faulkner, L. R. Electrochemical Methods: Fundamentals and Applications; Wiley, 2000.

(2) Dickinson, E. J. F.; Compton, R. G. Influence of the diffuse double layer on steady-state voltammetry. J. Electroanal. Chem. 2011, 661, 198-212.

(3) Chapman, D. L. LI. A contribution to the theory of electrocapillarity. Philos. Mag. 1913, 25, 475-481.

(4) Gouy, M. Sur la constitution de la charge électrique à la surface d'un électrolyte. J. Phys. Theor. Appl. 1910, 9, 457-468.

(5) Bazant, M. Z.; Thornton, K.; Ajdari, A. Diffuse-charge dynamics in electrochemical systems. Phys. Rev. E: Stat., Nonlinear, Soft Matter Phys. 2004, 70, 021506.

(6) Bazant, M. Z.; Storey, B. D.; Kornyshev, A. A. Double Layer in Ionic Liquids: Overscreening versus Crowding. Phys. Rev. Lett. 2011, $106,046102$.

(7) Hayes, R.; Borisenko, N.; Tam, M. K.; Howlett, P. C.; Endres, F.; Atkin, R. Double Layer Structure of Ionic Liquids at the $\mathrm{Au}(111)$ Electrode Interface: An Atomic Force Microscopy Investigation. J. Phys. Chem. C 2011, 115, 6855-6863.

(8) Fedorov, M. V.; Kornyshev, A. A. Ionic Liquids at Electrified Interfaces. Chem. Rev. 2014, 114, 2978-3036.

(9) Hayes, R.; Warr, G. G.; Atkin, R. Structure and Nanostructure in Ionic Liquids. Chem. Rev. 2015, 115, 6357-6426.

(10) Favaro, M.; Jeong, B.; Ross, P. N.; Yano, J.; Hussain, Z.; Liu, Z.; Crumlin, E. J. Unravelling the electrochemical double layer by direct probing of the solid/liquid interface. Nat. Commun. 2016, 7, 12695.

(11) Watanabe, M.; Thomas, M. L.; Zhang, S.; Ueno, K.; Yasuda, T.; Dokko, K. Application of Ionic Liquids to Energy Storage and Conversion Materials and Devices. Chem. Rev. 2017, 117, 7190-7239.

(12) Eftekhari, A. Supercapacitors utilising ionic liquids. Energy Storage Mater. 2017, 9, 47-69.

(13) Welton, T. Ionic liquids: a brief history. Biophys. Rev. 2018, 10, 691-706.

(14) Wang, Y.-L.; Li, B.; Sarman, S.; Mocci, F.; Lu, Z.-Y.; Yuan, J.; Laaksonen, A.; Fayer, M. D. Microstructural and Dynamical Heterogeneities in Ionic Liquids. Chem. Rev. 2020, 120, 5798-5877.

(15) Tian, Y.; Zeng, G.; Rutt, A.; Shi, T.; Kim, H.; Wang, J.; Koettgen, J.; Sun, Y.; Ouyang, B.; Chen, T.; Lun, Z.; Rong, Z.; Persson, K.; Ceder, G. Promises and Challenges of Next-Generation "Beyond Li-ion" Batteries for Electric Vehicles and Grid Decarbonization. Chem. Rev. 2021, 121, 1623-1669.

(16) Begić, S.; Chen, F.; Jónsson, E.; Forsyth, M. Overscreening and crowding in electrochemical ionic liquid systems. Phys. Rev. Mater. 2019, 3, 095801.

(17) Lynden-Bell, R. M.; Frolov, A. I.; Fedorov, M. V. Electrode screening by ionic liquids. Phys. Chem. Chem. Phys. 2012, 14, 26932701.

(18) Atkin, R.; Borisenko, N.; Drüschler, M.; El Abedin, S. Z.; Endres, F.; Hayes, R.; Huber, B.; Roling, B. An in situSTM/AFM and impedance spectroscopy study of the extremely pure 1-butyl-1methylpyrrolidinium tris(pentafluoroethyl)trifluorophosphate/ $\mathrm{Au}(111)$ interface: potential dependent solvation layers and the herringbone reconstruction. Phys. Chem. Chem. Phys. 2011, 13, 6849-6857.

(19) Perkin, S.; Crowhurst, L.; Niedermeyer, H.; Welton, T.; Smith, A. M.; Gosvami, N. N. Self-assembly in the electrical double layer of ionic liquids. Chem. Commun. 2011, 47, 6572-6574. 
(20) Storey, B. D.; Bazant, M. Z. Effects of electrostatic correlations on electrokinetic phenomena. Phys. Rev. E: Stat., Nonlinear, Soft Matter Phys. 2012, 86, 056303.

(21) Kilic, M. S.; Bazant, M. Z.; Ajdari, A. Steric effects in the dynamics of electrolytes at large applied voltages. I. Double-layer charging. Phys. Rev. E: Stat., Nonlinear, Soft Matter Phys. 2007, 75, 021502.

(22) Kilic, M. S.; Bazant, M. Z.; Ajdari, A. Steric effects in the dynamics of electrolytes at large applied voltages. II. Modified Poisson-NernstPlanck equations. Phys. Rev. E: Stat., Nonlinear, Soft Matter Phys. 2007, $75,021503$.

(23) Lockett, V.; Horne, M.; Sedev, R.; Rodopoulos, T.; Ralston, J. Differential capacitance of the double layer at the electrode/ionic liquids interface. Phys. Chem. Chem. Phys. 2010, 12, 12499-12512.

(24) Lockett, V.; Sedev, R.; Ralston, J.; Horne, M.; Rodopoulos, T. Differential Capacitance of the Electrical Double Layer in ImidazoliumBased Ionic Liquids: Influence of Potential, Cation Size, and Temperature. J. Phys. Chem. C 2008, 112, 7486-7495.

(25) Zheng, J.; Moganty, S. S.; Goonetilleke, P. C.; Baltus, R. E.; Roy, D. A Comparative Study of the Electrochemical Characteristics of $[$ Emim +$][\mathrm{BF} 4-]$ and $[\mathrm{Bmim}+][\mathrm{BF} 4-]$ Ionic Liquids at the Surfaces of Carbon Nanotube and Glassy Carbon Electrodes. J. Phys. Chem. C 2011, 115, 7527-7537.

(26) Atkin, R.; Borisenko, N.; Drüschler, M.; Endres, F.; Hayes, R.; Huber, B.; Roling, B. Structure and dynamics of the interfacial layer between ionic liquids and electrode materials. J. Mol. Liq. 2014, 192, 44-54.

(27) Drüschler, M.; Huber, B.; Roling, B. On Capacitive Processes at the Interface between 1-Ethyl-3-methylimidazolium tris(pentafluoroethyl)trifluorophosphate and $\mathrm{Au}(111)$. J. Phys. Chem. C 2011, 115, 6802-6808.

(28) Perez-Martinez, C. S.; Perkin, S. Surface forces generated by the action of electric fields across liquid films. Soft Matter 2019, 15, 42554265.

(29) Uysal, A.; Zhou, H.; Feng, G.; Lee, S. S.; Li, S.; Cummings, P. T.; Fulvio, P. F.; Dai, S.; McDonough, J. K.; Gogotsi, Y.; Fenter, P. Interfacial ionic 'liquids': connecting static and dynamic structures. J. Phys.: Condens. Matter 2014, 27, 032101.

(30) Chu, M.; Miller, M.; Douglas, T.; Dutta, P. Ultraslow Dynamics at a Charged Silicon-Ionic Liquid Interface Revealed by X-ray Reflectivity. J. Phys. Chem. C 2017, 121, 3841-3845.

(31) Collins, L.; Jesse, S.; Kilpatrick, J. I.; Tselev, A.; Varenyk, O.; Okatan, M. B.; Weber, S. A. L.; Kumar, A.; Balke, N.; Kalinin, S. V.; Rodriguez, B. J. Probing charge screening dynamics and electrochemical processes at the solid-liquid interface with electrochemical force microscopy. Nat. Commun. 2014, 5, 3871.

(32) Collins, L.; Kilpatrick, J. I.; Kalinin, S. V.; Rodriguez, B. J. Towards nanoscale electrical measurements in liquid by advanced KPFM techniques: a review. Rep. Prog. Phys. 2018, 81, 086101.

(33) Weingarth, D.; Foelske-Schmitz, A.; Wokaun, A.; Kötz, R. In situ electrochemical XPS study of the Pt/[EMIM][BF4] system. Electrochem. Commun. 2011, 13, 619-622.

(34) Camci, M. T.; Aydogan, P.; Ulgut, B.; Kocabas, C.; Suzer, S. XPS enables visualization of electrode potential screening in an ionic liquid medium with temporal- and lateral-resolution. Phys. Chem. Chem. Phys. 2016, 18, 28434-28440.

(35) Aydogan Gokturk, P.; Ulgut, B.; Suzer, S. DC Electrowetting of Nonaqueous Liquid Revisited by XPS. Langmuir 2018, 34, 7301-7308.

(36) Aydogan Gokturk, P.; Ulgut, B.; Suzer, S. AC Electrowetting Modulation of Low-Volatile Liquids Probed by XPS: Dipolar vs Ionic Screening. Langmuir 2019, 35, 3319-3326.

(37) Uzundal, C. B.; Sahin, O.; Gokturk, P. A.; Wu, H.; Mugele, F.; Ulgut, B.; Suzer, S. X-ray Photoelectron Spectroscopy with Electrical Modulation Can Be Used to Probe Electrical Properties of Liquids and Their Interfaces at Different Stages. Langmuir 2019, 35, 16989-16999.

(38) Uzundal, C. B.; Gokturk, P. A.; Suzer, S.; Ulgut, B. CoarseGrained Electrostatic Model Including Ion-Pairing Equilibrium That Explains DC and AC X-ray Photoelectron Spectroscopy Measurements on Ionic Liquids. J. Phys. Chem. C 2019, 123, 13192-13200.
(39) Aydogan Gokturk, P.; Taner Camci, M.; Suzer, S. Lab-based operando $\mathrm{x}$-ray photoelectron spectroscopy for probing low-volatile liquids and their interfaces across a variety of electrosystems. J. Vac. Sci. Technol., A 2020, 38, 040805.

(40) Zhang, S.; Brahim, S.; Maat, S. High-voltage operation of binderfree CNT supercapacitors using ionic liquid electrolytes. J. Mater. Res. 2018, 33, 1179-1188.

(41) Aydogan Gokturk, P.; Salzner, U.; Nyulászi, L.; Ulgut, B.; Kocabas, C.; Suzer, S. XPS-evidence for in-situ electrochemicallygenerated carbene formation. Electrochim. Acta 2017, 234, 37-42.

(42) Aydogan Gokturk, P.; Donmez, S. E.; Ulgut, B.; Türkmen, Y. E.; Suzer, S. Optical and XPS evidence for the electrochemical generation of an N-heterocyclic carbene and its CS2 adduct from the ionic liquid [bmim][PF6]. New J. Chem. 2017, 41, 10299-10304.

(43) Noori, A.; El-Kady, M. F.; Rahmanifar, M. S.; Kaner, R. B.; Mousavi, M. F. Towards establishing standard performance metrics for batteries, supercapacitors and beyond. Chem. Soc. Rev. 2019, 48, 12721341.

(44) RC Differentiator. https://www.electronics-tutorials.ws/rc/rcdifferentiator.html (accessed January 2, 2021).

(45) Greco, F.; Shin, S.; Williams, F. J.; Heller, B. S. J.; Maier, F.; Steinrück, H. P. Potential Screening at Electrode/Ionic Liquid Interfaces from In Situ X-ray Photoelectron Spectroscopy. ChemistryOpen 2019, 8, 1365-1368.

(46) Gebbie, M. A.; Valtiner, M.; Banquy, X.; Fox, E. T.; Henderson, W. A.; Israelachvili, J. N. Ionic liquids behave as dilute electrolyte solutions. Proc. Natl. Acad. Sci. U. S. A. 2013, 110, 9674-9679.

(47) Gebbie, M. A.; Smith, A. M.; Dobbs, H. A.; Lee, A. A.; Warr, G. G.; Banquy, X.; Valtiner, M.; Rutland, M. W.; Israelachvili, J. N.; Perkin, S.; Atkin, R. Long range electrostatic forces in ionic liquids. Chem. Commun. 2017, 53, 1214-1224.

(48) Lee, A. A.; Perez-Martinez, C. S.; Smith, A. M.; Perkin, S. Underscreening in concentrated electrolytes. Faraday Discuss. 2017, 199, 239-259.

(49) Tokuda, H.; Hayamizu, K.; Ishii, K.; Susan, M. A. B. H.; Watanabe, M. Physicochemical Properties and Structures of Room Temperature Ionic Liquids. 2. Variation of Alkyl Chain Length in Imidazolium Cation. J. Phys. Chem. B 2005, 109, 6103-6110.

(50) Lee, A. A.; Vella, D.; Perkin, S.; Goriely, A. Are RoomTemperature Ionic Liquids Dilute Electrolytes? J. Phys. Chem. Lett. 2015, 6, 159-163.

(51) Noda, A.; Hayamizu, K.; Watanabe, M. Pulsed-Gradient SpinEcho $1 \mathrm{H}$ and 19F NMR Ionic Diffusion Coefficient, Viscosity, and Ionic Conductivity of Non-Chloroaluminate Room-Temperature Ionic Liquids. J. Phys. Chem. B 2001, 105, 4603-4610.

(52) de Levie, R. On porous electrodes in electrolyte solutions: I. Capacitance effects. Electrochim. Acta 1963, 8, 751-780.

(53) Mirzadeh, M.; Gibou, F.; Squires, T. M. Enhanced Charging Kinetics of Porous Electrodes: Surface Conduction as a Short-Circuit Mechanism. Phys. Rev. Lett. 2014, 113, 097701. 